\title{
Temperate forest bird communities associated with a historic mining impact area: do tailing remnant effects modify their structure?
}

\author{
Katia Lemus ${ }^{1}$, José Fernando Villaseñor-Gómez ${ }^{1,2}$, Francisco Roberto Pineda-Huerta ${ }^{1}$ \\ \& Javier Salgado-Ortiz ${ }^{1}$
}

1 Laboratorio de Investigación en Ornitología, Facultad de Biología, Universidad Michoacana de San Nicolás de Hidalgo, Ciudad Universitaria, Morelia, Michoacán, Mexico.

2 Corresponding author: jfvillasenorg@hotmail.com

Received on 21 February 2019. Accepted on 04 June 2019.

\begin{abstract}
Birds contribute to the stability of ecosystems and represent a tool used to evaluate a variety of anthropogenic impacts. The area known as El Oro-Tlalpujahua Mining District in central Mexico was subjected to significant environmental impacts as a result of ore extraction, including profound habitat transformations, landscape changes, and the accumulation of potentially toxic elements in their tailings (favoring its bioavailability and dispersion). After more than 60 years without extractive activities, there is no knowledge on extant remaining impacts on biological communities. Assuming the presence of negative impacts on birds, we compared the composition and abundance of bird communities in two locations, representing a site without exposure to tailings $\left(S_{1}\right)$ and another one with tailings deposition $\left(S_{2}\right)$. From June 2014 to June 2015, we recorded 2828 individuals of 108 avian species in 369 point counts $\left(S_{1}=91, S_{2}=95\right)$. The Chao1 indicator suggested we recorded $96 \%$ of the species present. We found a high similarity in the general composition and abundance of bird species between communities $(>85 \%)$. However, there were significant differences in the abundances of 18 species ( 9 of them higher in the control site); these differences might result from differential effects of potentially toxic elements on functional groups (such as feeding guilds), resource availability, as well as other factors not accounted for. Historically, mining activities in the area generated significant changes in the structure and composition of the forest, and disrupted ecological processes. Despite the fact that current conditions appear favorable to the relative stability of the bird community, specific physiological effects on some species of birds sixty years after the cessation of mineral extraction could occur. Further studies on physiological performance and the effects of potentially toxic elements on local birds could unveil unknown effects at the individual level.
\end{abstract}

KEY-WORDS: avian communities, diversity, El Oro-Tlalpujahua Mining District, mining tailings, remnant effects.

\section{INTRODUCTION}

Information on the diversity and abundance of species in communities represent the basis for assessing the quality of their environment. Through monitoring it is possible to evaluate changes associated with different causal factors; comparisons on the occurrence of species in different environments, and the characterization of their relative abundance is often presented as supporting evidence (Balmer 2002).

Disturbance processes generated by human activities involve habitat changes through the modification of land use for productive activities (e.g., agriculture and livestock), urban development, and mining activities, with the resulting effects on soil and water (Manson \& JardelPeláez 2009). These events at the landscape level modify the structure of vegetation and generate successional processes that promote changes in the structure and composition of animal communities (Pickett \& White 1985). In birds, these types of changes have been described previously by Ugalde-Lezama et al. (2012) and Manson \& Jardel-Peláez (2009), who found the simplification of forest structure related to the decrease in the composition of bird communities.

To evaluate anthropogenic impacts on wildlife, it is desirable to have an indicator of the intensity and extent of the impacts; if significant, they might be reflected in changes in the composition and/or abundance of species at the community level. At the population level, impacts may be reflected in changes in survival rates or reproductive success of species, or changes in their distribution (Altaf et al. 2018, Mahmoud \& Gan 2018, $\mathrm{Xu}$ et al. 2018). Responses at the individual level are the most sensitive and usually have been assessed through changes in physical and physiological conditions (e.g., height, weight, condition index, quantity of fat reserves) 
(Pérez-Tris 1999). Few studies have explored the response of birds to anthropogenic impacts resulting from mining at the community level; most have focused on particular species (e.g., Garitano-Zavala et al. 2010, Rubio et al. 2016), or ecological settings (e.g., Ouboter et al. 1999, Eagles-Smith et al. 2016).

Several authors recognize the need for environmental monitoring from geological, ecological, and public health perspectives (Boulet \& Larocque 1988, Perotti et al. 2017). Reclamation mining sites have sometimes been perceived as sites potentially important for biodiversity (Batty 2005). Mines that have operated for centuries are the source of pollutants that remain stored in tailings or the bottom of reservoirs, and their ecological effects in most cases have not been determined (Kossoff et al. 2014). Globally, estimations of mercury released to the environment as a byproduct of the amalgamation for recovery of gold and silver indicate that there has been over 260,000 tons released between 1550 and 1930 (Lacerda 1997). Furthermore, for mining sites that historically ceased production and left a legacy of ecological impact, little is known about the span and intensity of their impacts (Balistrieri et al. 2002, Eisler 2004, Cristol et al. 2008, Ventakeswarlu et al. 2016). Some studies have addressed aspects of geodynamics, bioavailability and transfer of elements in mine tailings, which are potentially toxic elements derived from mining runoff and water currents (e.g., Rösner 1988, Perotti et al. 2017), as well as on soil and vegetation (O'Sullivan et al. 1999, Jacob \& Otte 2004, Struckhoff et al. 2013), aquatic and terrestrial animals (such as benthic invertebrates, springtail insects, fish, amphibians, reptiles and birds), remediation, and ecological restoration (Lefcort et al. 1988, GonçalvesRodriguez \& Shraft 2001, Lock et al. 2003, Cristol et al. 2008, Márquez-Ferrando 2008). Data from historically important mining areas in the world is scarce, and there is no documented information on possible remnant effects in reference to wildlife.

The Oro-Tlalpujahua Mining District (OTMD) in central Mexico has been the site of ecological changes associated with the settlement of a very important center of gold and silver ore extraction, from the middle $19^{\text {th }}$ to early $20^{\text {th }}$ century (Corona-Chávez \& Uribe-Salas 2009). Mina Dos Estrellas was an exceptional settlement in its time, whose establishment and operation with major infrastructure caused the almost complete deforestation of the original forests, and led to the creation of roads, landscape alteration, and the accumulation of waste materials from ore extraction, among others. As a result of continued activities, the area gradually accumulated tailings of momentous volume. These elements have defined the environmental history of the region, and have stimulated interest in understanding the long term consequences of disturbance in the area (Corona-Chávez et al. 2010).
During the 65 years after cessation of activities in Mina Dos Estrellas, the region has experienced ecological succession that led to the reestablishment of secondary temperate forest made up of tolerant and pioneer tree species that survived the disturbance period on impoverished soil conditions (Muñiz-Castro 2008).

Because of the demands of wood and other materials for the construction and maintenance of mine galleries, the surrounding areas, and even those far away were also overexploited (Corona-Chávez \& Uribe-Salas 2009). While the area was subjected to a strong mining impact, at the end of the mine's active life some nearby areas remained free from the effects of ore wastes, deforestation, agriculture and cattle grazing. These areas offer the possibility of investigating if some remnant effects derived from mining in the past are maintained and affect bird communities. Considering the possibilities of extant impacts, we analyzed and compared the richness and abundance of forest bird communities inhabiting tailings sites (abandoned approximately 65 years ago) and sites free from mine wastes, in order to determine differences that might be indicative of remnant impacts on the avifauna. Facing a possible scenario of intense and prolonged impact induced by the bioavailability of potentially toxic elements in tailings, we expected bird communities away from tailings to be more diverse and have higher abundance at least for the most common species, in comparison to the polluted area.

\section{METHODS}

\section{Study area}

The OTMD is situated in the limits of the states of Michoacan and Mexico $\left(19^{\circ} 18^{\prime} \mathrm{N} ; 100^{\circ} 09^{\prime} \mathrm{W}\right.$; Fig. 1) (Nieto-Monroy 2007), as part of the TransMexican Volcanic Belt. At an elevation ranging from 2600 to $2850 \mathrm{~m}$ a.s.l., $45 \%$ of its surface is covered by secondary forest of Cedars (Cupressus lusitanica), Junipers (Juniperus deppeana), oaks (Quercus spp.), and pines (Pinus spp.). Its climate is temperate sub-humid with rainfall in summer $(800-1100 \mathrm{~mm}$ per year), and the soils are mainly represented by andosols and luvisols (INEGI 2009). The OTMD area is adjacent to the polygons of the Monarch Butterfly Biosphere Reserve (MBBR) (Coronado-Martínez 2016). Although OTDM is not part of the reserve, it has had influences within the protected area because of historical extraction of materials in the past and tourism activities in the present (Ramírez-Ramírez 2001, SEMARNAT 2001, Coronado-Martínez 2016). Due to the limited and specific features of the area, the study sites are included only in one site, without replicates. 


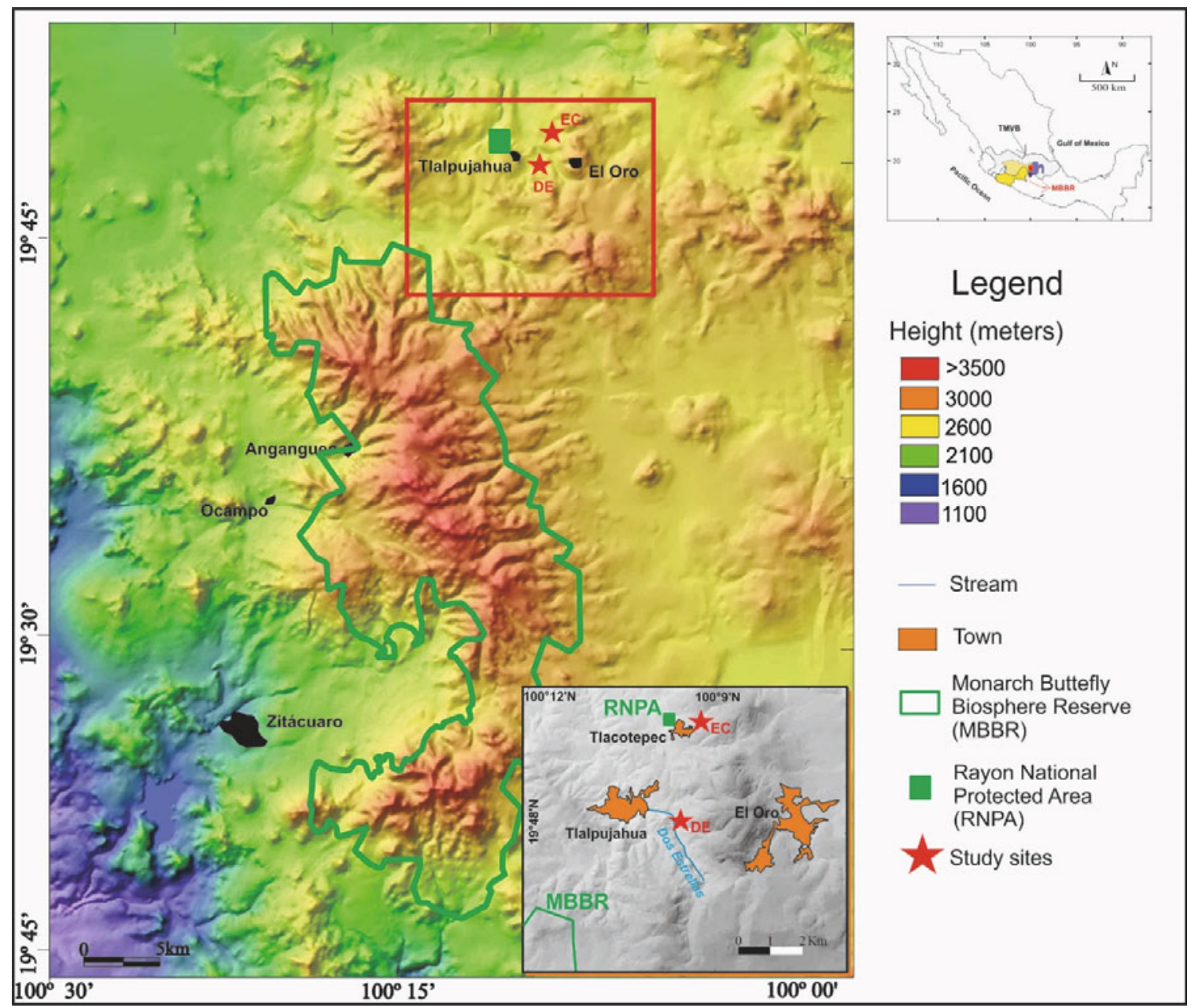

Figure 1. The Oro-Tlalpujahua Mining District study area in central Mexico $\left(E C=E l\right.$ Castillo, $S_{1} ; D E=$ Mina Dos Estrellas, $S_{2}$ ).

\section{Bird sampling}

Bird sampling was carried out in two study sites: a) a control site far from tailings (El Castillo, Tlacotepec, $\mathrm{S}_{1} ; 19.822^{\circ} \mathrm{N} ;-100.145^{\circ} \mathrm{W}, 2750 \mathrm{~m}$ a.s.l.; Fig. $2 \mathrm{~A}$ ) and b) a tailings site (Mina Dos Estrellas, Tlalpujahua, $\mathrm{S}_{2}$; $19.793^{\circ} \mathrm{N} ; 100.156^{\circ} \mathrm{W}, 2648 \mathrm{~m}$ a.s.l.; Fig. 2B), both within the municipality of Tlalpujahua, Michoacan. Vegetation in both sites resulted from a natural secondary succession process and have similar structure and composition (Osuna-Vallejo et al. 2016). To determine the composition and abundance of bird communities, every month from June 2014 to June 2015, we conducted a total of 369 point counts $(10 \mathrm{~min}$ ) (169 in the control site $S_{1}$ and 200 in the tailings site $S_{2}$ ), located randomly every $200 \mathrm{~m}$ along independent paths, in which we registered individuals detected visually and acoustically (by songs or calls) within a fixed $50 \mathrm{~m}$ radius (to avoid bias due to detectability) (Hutto et al. 1986, Buskirk \& McDonald 1995). The taxonomic arrangement adopted here was that proposed by the American Ornithological Society (AOS 2017), while the assignment of species to seasonality categories was based on our own experience and Howell \& Webb (2005). Species considered in any concern category were defined according to Norma Oficial Mexicana NOM-059-SEMARNAT-2010, where native species of wild flora and fauna in Mexico considered in any conservation risk are listed (SEMARNAT 2010).

\section{Data analysis}

We estimated the relative abundance and frequency of occurrence of bird species by site. The former was expressed by the number of individuals in 100 point counts, and the latter was evaluated through the percentage of counts where the species was recorded, which can reflect the detection probability of the species (Hutto et al. 1986).

We used the Completeness Index (Chao 1 estimator) to make a prediction of the expected species in the community based on our sampling (Chao et al. 2005). For each site we generated species accumulation curves to ensure sampling effort was adequate and to compare richness among sites (Colwell \& Coddington 1994). These analyses were performed in EstimateS 9.1.0 (Colwell 2013).

In order to compare the similarity of communities 

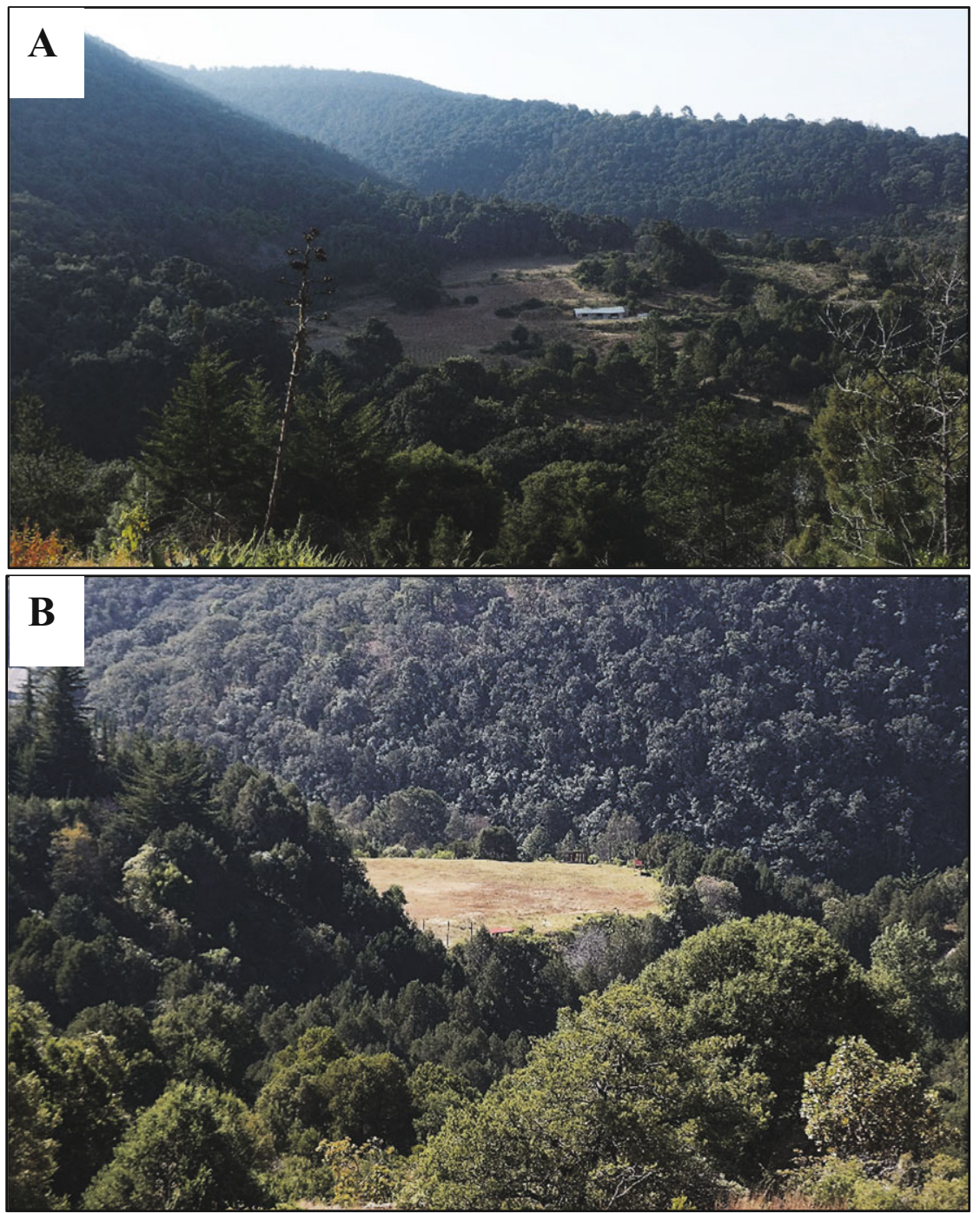

Figure 2. Control site, El Castillo, Tlacotepec, Michoacan (A); mining site, Mina Dos Estrellas (B), Photo authors: K.I. LemusRamírez (A) and J.F. Villaseñor-Gómez (B).

between sites, we computed the qualitative Sorensen index and the quantitative Morisita-Horn index using Excel 2013. The former index is based on species presence/ absence data, and indicates the composition resemblance of the communities; while the latter considers the number of individuals registered for each one of the species (Badii et al. 2007). To evaluate differences in species' abundance between sampling sites we applied a nonparametric Mann-Whitney U test using IBM SPSS Statistics 20.0). We also used an Analysis of Similarity (ANOSIM) to compare the degree of correspondence in the composition of communities (sensu Blake 2007, Edwards et al. 2011); as this method evaluates a dissimilarity matrix, values of $R$ closer to zero reflect very similar communities, and values close to unity reflect significant differences between the communities being compared (PAST version $2.17 \mathrm{c}$ ). In order to gain further information about the species' contribution to the differences between communities, we applied a Similarity Percentage Analysis SIMPER (PAST version $2.17 \mathrm{c}$ ), that breaks up the contribution of each one of the species to the similarity observed between communities, and defines the most important species responsible for the observed pattern (Clarke 1993).

\section{RESULTS}

From June 2014 to June 2015 we conducted a total of 369 point counts (169 in the control site $S_{1}$ and 200 in the tailings site $S_{2}$ ), and detected a total of 4364 individuals $\left(S_{1}=2043, S_{2}=2321\right)$ from 108 species and 30 families $\left(S_{1}=97, S_{2}=91\right) ; 85$ are resident, 20 are winter visitors, two are considered transitory, and one is an introduced resident species (Table 1). We identified ten functional or guild groups (groups of species in a community that exploit the same set of resources in a similar manner, but are not necessarily closely related taxonomically). In the two study sites the insectivorous guild was the most abundant $\left(S_{1}=48 \%\right.$ species, $35 \%$ individuals; $S_{2}=51 \%$ species, $35 \%$ individuals), followed by the omnivorous guild $\left(S_{1}\right.$ 
$=19 \%$ species, $30 \%$ individuals; $S_{2}=20 \%$ species, $23 \%$ individuals). In general, the communities showed very similar functional structure (species: $\chi^{2}=1.928, P=0.993$; individuals: $\chi^{2}=2.856, P=0.970$; Fig. 3).

Species accumulation curves exhibited an asymptotic behavior suggesting an adequate sampling effort for the detection of most species in the area. The Completeness Index $(\mathrm{CI}=$ Sobs/Sest $)$ that is computed along with $\log$-linear $95 \%$ confidence intervals (CI), indicated the recording of $96 \%$ of species for the OTMD region. The number of estimated species for the control site $S_{1}$ was $83 \%$ of the species recorded (117 estimated species, IC = 104-157 species), meanwhile for the tailings site $S_{2}$, the estimated species corresponded to $93 \%$ of the detected ones (98 estimated species, $\mathrm{IC}=93-116$ species). Eighteen species were exclusive to the control site $S_{1}$, ten were exclusive to the tailings site $S_{2}$, and 88 species were present in both communities (Table 1). All exclusive species for each site were rare and infrequently recorded.

With respect to their relative abundances, our results showed that at the control site $S_{1}$, Ptiliogonys cinereus (Gray Silky-flycatcher), Hylocharis leucotis (White-eared Hummingbird), Spizella passerina (Chipping Sparrow), Regulus calendula (Ruby-crowned Kinglet), and Turdus migratorius (American Robin) were the most abundant species. Correspondingly, at the tailings site $S_{2}$, the species with the greatest relative abundance were Setophaga coronata (Yellow-rumped Warbler), P. cinereus, H. leucotis,
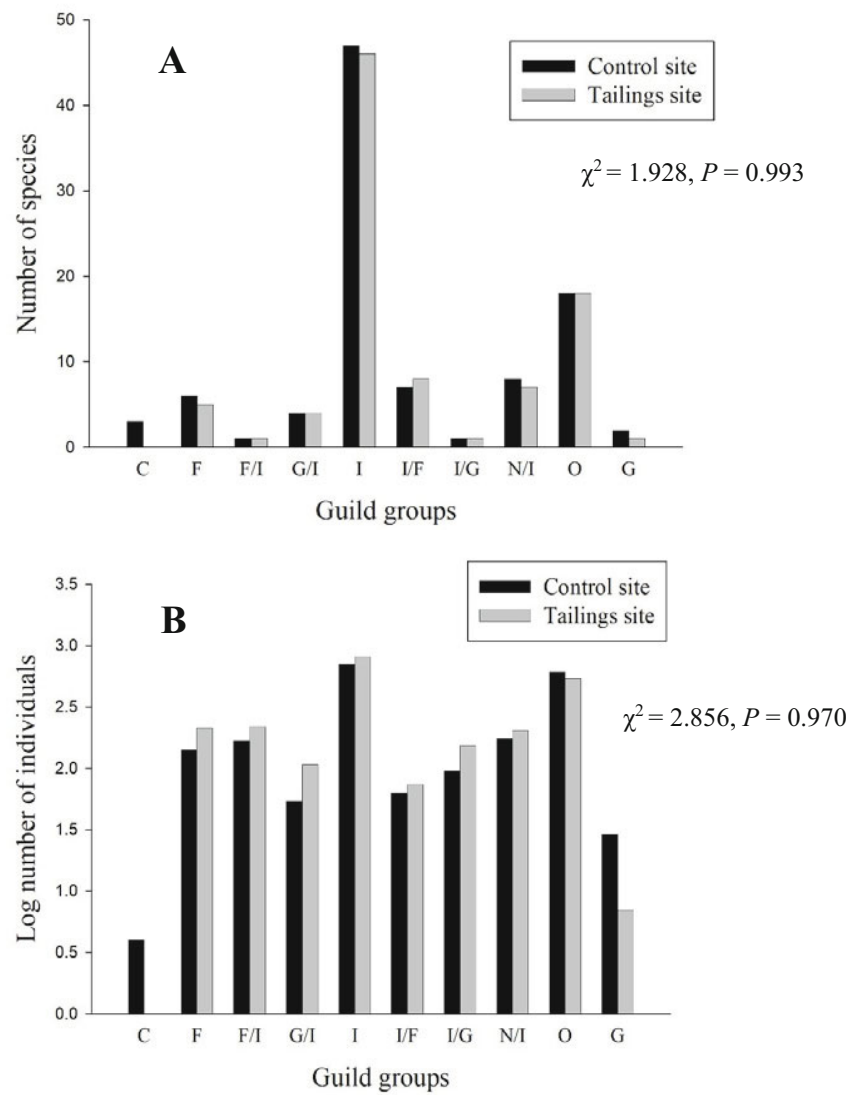

Figure 3. Bar chart of (A) number of species and (B) individuals belonging to different guilds at two sites within the El Oro-Tlalpujahua Mining District area. Legend: C: Carnivorous, F: Frugivorous, G: Granivorous, I: Insectivorous, $\mathrm{N}$ : Nectarivorous, O: Omnivorous.

Table 1. Seasonal status and relative abundances of bird species recorded in a control site and a mining site at El OroTlalpujahua Mining District, central Mexico.

\begin{tabular}{|c|c|c|c|c|c|c|}
\hline \multirow{2}{*}{$\begin{array}{l}\text { Family } \\
\text { Species }\end{array}$} & \multirow[t]{2}{*}{ Common name ${ }^{a}$} & \multirow{2}{*}{$\begin{array}{c}\text { Seasonal } \\
\text { status }^{\mathbf{b}}\end{array}$} & \multicolumn{2}{|c|}{$\begin{array}{c}\text { El Castillo } \\
\left(S_{1} \text { Control site }\right)\end{array}$} & \multicolumn{2}{|c|}{$\begin{array}{l}\text { Mina Dos Estrellas } \\
\left.\text { ( } \mathrm{S}_{2} \text { Mining site }\right)\end{array}$} \\
\hline & & & FRE $S_{1}{ }^{c}$ & $\mathrm{ABUS}_{1}{ }^{\mathrm{d}}$ & FRE $S_{2}{ }^{\mathrm{c}}$ & $\mathrm{ABU} \mathrm{S_{2 } { } ^ { \mathrm { d } }}$ \\
\hline \multicolumn{7}{|l|}{ Odontophoridae } \\
\hline Colinus virginianus & Northern Bobwhite & PR & 0.59 & 1.18 & NR & NR \\
\hline \multicolumn{7}{|l|}{ Columbidae } \\
\hline Patagioenas fasciata & Band-tailed Pigeon & PR & 0.59 & 0.59 & NR & NR \\
\hline Columbina inca & Inca Dove & PR & 5.92 & 14.20 & 2.00 & 5.25 \\
\hline \multicolumn{7}{|l|}{ Cuculidae } \\
\hline Geococcyx californianus & Greater Roadrunner & PR & 0.59 & 0.59 & NR & NR \\
\hline \multicolumn{7}{|l|}{ Caprimulgidae } \\
\hline Antrostomus arizonae & Mexican Whip-poor-will & PR & NR & NR & 0.50 & 0.75 \\
\hline \multicolumn{7}{|l|}{ Trochilidae } \\
\hline Colibri thalassinus & Mexican Violetear & PR & 4.14 & 4.73 & 4.50 & 6.75 \\
\hline Eugenes fulgens & Rivoli's Hummingbird & PR & 4.73 & 5.92 & 4.00 & 6.75 \\
\hline Lampornis clemenciae & Blue-throated Humming. & PR & 1.78 & 2.96 & 2.50 & 3.75 \\
\hline Archilochus colubris & Ruby-throated Humming. & $\mathrm{TR}$ & 1.18 & 1.18 & NR & NR \\
\hline Selasphorus platycercus & Broad-tailed Hummingbird & PR & 1.78 & 1.78 & 0.50 & 0.75 \\
\hline Selasphorus rufus & Rufous Hummingbird & VI & 0.59 & 0.59 & 0.50 & 0.75 \\
\hline Amazilia beryllina & Berylline Hummingbird & PR & 0.59 & 1.18 & 3.00 & 6.00 \\
\hline
\end{tabular}




\begin{tabular}{|c|c|c|c|c|c|c|}
\hline \multirow{2}{*}{$\begin{array}{l}\text { Family } \\
\text { Species } \\
\end{array}$} & \multirow[t]{2}{*}{ Common name ${ }^{a}$} & \multirow{2}{*}{$\begin{array}{l}\text { Seasonal } \\
\text { status }^{\mathrm{b}}\end{array}$} & \multicolumn{2}{|c|}{$\begin{array}{c}\text { El Castillo } \\
\left(\mathrm{S}_{1} \text { Control site }\right) \\
\end{array}$} & \multicolumn{2}{|c|}{$\begin{array}{c}\text { Mina Dos Estrellas } \\
\left(\mathrm{S}_{2} \text { Mining site }\right) \\
\end{array}$} \\
\hline & & & FRE $S_{1}{ }^{c}$ & $\mathrm{ABU} S_{1}{ }^{\mathrm{d}}$ & FRE $S_{2}{ }^{\mathrm{c}}$ & $\mathrm{ABU} \mathrm{S}_{2}^{\mathrm{d}}$ \\
\hline Hylocharis leucotis & White-eared Hummingbird & PR & 63.31 & 85.21 & 59.50 & 128.25 \\
\hline \multicolumn{7}{|l|}{ Accipitridae } \\
\hline Accipiter cooperii ${ }^{* *}$ & Cooper's Hawk & VI & 1.18 & 1.18 & NR & NR \\
\hline Buteo jamaicensis & Red-tailed Hawk & PR & 0.59 & 0.59 & NR & NR \\
\hline \multicolumn{7}{|l|}{ Trogonidae } \\
\hline Trogon mexicanus & Mountain Trogon & PR & 4.14 & 5.92 & NR & NR \\
\hline \multicolumn{7}{|l|}{ Picidae } \\
\hline Melanerpes formicivorus & Acorn Woodpecker & PR & 9.47 & 15.98 & 18.50 & 64.50 \\
\hline Picoides scalaris & Ladder-backed Woodpeck. & PR & 6.51 & 6.51 & 4.50 & 6.75 \\
\hline Picoides villosus & Hairy Woodpecker & PR & 3.55 & 4.14 & 3.50 & 5.25 \\
\hline Colaptes auratus & Northern Flicker & PR & 5.33 & 5.33 & 0.50 & 0.75 \\
\hline \multicolumn{7}{|l|}{ Tyrannidae } \\
\hline Mitrephanes phaeocercus & Tufted Flycatcher & PR & 8.28 & 8.88 & 5.50 & 10.50 \\
\hline Contopus pertinax & Greater Pewee & PR & 14.79 & 15.98 & 12.00 & 18.00 \\
\hline Empidonax affinis & Pine Flycatcher & PR & 0.59 & 0.59 & NR & NR \\
\hline Empidonax difficilis & Pacific-slope Flycatcher & VI & 1.18 & 1.18 & NR & NR \\
\hline Empidonax occidentalis & Cordilleran Flycatcher & PR & 12.43 & 13.02 & 10.00 & 17.25 \\
\hline Empidonax fulvifrons & Buff-breasted Flycatcher & PR & 1.18 & 1.18 & 2.50 & 4.50 \\
\hline Sayornis nigricans & Black Phoebe & PR & NR & NR & 0.50 & 1.50 \\
\hline Sayornis saya & Say's Phoebe & VI & 0.59 & 1.18 & NR & NR \\
\hline Pyrocephalus rubinus & Vermilion Flycatcher & PR & 2.96 & 5.33 & 0.50 & 0.75 \\
\hline Myiarchus tuberculifer & Dusky-capped Flycatcher & PR & 1.78 & 2.37 & 1.50 & 3.00 \\
\hline Tyrannus vociferans & Cassin's Kingbird & PR & 5.92 & 7.69 & 2.00 & 3.75 \\
\hline \multicolumn{7}{|l|}{ Tityridae } \\
\hline Pachyramphus aglaiae & Rose-throated Becard & PR & 0.59 & 0.59 & 0.50 & 1.50 \\
\hline \multicolumn{7}{|l|}{ Vireonidae } \\
\hline Vireo huttoni & Hutton's Vireo & PR & 5.92 & 7.10 & 5.50 & 9.00 \\
\hline Vireo cassinii & Cassin's Vireo & VI & 4.73 & 5.92 & 2.50 & 4.50 \\
\hline Vireo plumbeus & Plumbeous Vireo & PR & 0.59 & 0.59 & 0.50 & 0.75 \\
\hline Vireo gilvus & Warbling Vireo & PR & 0.59 & 0.59 & 0.50 & 0.75 \\
\hline \multicolumn{7}{|l|}{ Corvidae } \\
\hline Cyanocitta stelleri & Steller's Jay & PR & 5.33 & 20.71 & 1.00 & 5.25 \\
\hline Corvus corax & Common Raven & PR & NR & NR & 0.50 & 0.75 \\
\hline \multicolumn{7}{|l|}{ Hirundinidae } \\
\hline Tachycineta thalassina & Violet-green Swallow & PR & 0.59 & 0.59 & 1.50 & 2.75 \\
\hline \multicolumn{7}{|l|}{ Paridae } \\
\hline Poecile sclateri & Mexican Chickadee & PR & 3.55 & 8.28 & 1.00 & 4.50 \\
\hline Baeolophus wollweberi & Bridled Titmouse & PR & NR & NR & 0.50 & 1.50 \\
\hline \multicolumn{7}{|l|}{ Aegithalidae } \\
\hline Psaltriparus minimus & Bushtit & PR & 8.88 & 56.21 & 9.00 & 116.25 \\
\hline \multicolumn{7}{|l|}{ Sittidae } \\
\hline Sitta carolinensis & White-breasted Nuthatch & PR & 23.08 & 29.59 & 12.00 & 18.75 \\
\hline
\end{tabular}




\begin{tabular}{|c|c|c|c|c|c|c|}
\hline \multirow{2}{*}{$\begin{array}{l}\text { Family } \\
\text { Species } \\
\end{array}$} & \multirow[t]{2}{*}{ Common name ${ }^{a}$} & \multirow{2}{*}{$\begin{array}{c}\text { Seasonal } \\
\text { status }^{\mathrm{b}}\end{array}$} & \multicolumn{2}{|c|}{$\begin{array}{c}\text { El Castillo } \\
\left(S_{1} \text { Control site }\right)\end{array}$} & \multicolumn{2}{|c|}{$\begin{array}{l}\text { Mina Dos Estrellas } \\
\left(\mathrm{S}_{2} \text { Mining site }\right)\end{array}$} \\
\hline & & & FRE $S_{1}{ }^{c}$ & $A B U S_{1}^{d}$ & FRE $S_{2}{ }^{\mathrm{c}}$ & $\mathrm{ABU} \mathrm{S}{ }_{2}^{\mathrm{d}}$ \\
\hline \multicolumn{7}{|l|}{ Certhiidae } \\
\hline Certhia americana & Brown Creeper & PR & 0.59 & 0.59 & NR & NR \\
\hline \multicolumn{7}{|l|}{ Troglodytidae } \\
\hline Catherpes mexicanus & Canyon Wren & PR & 1.78 & 1.78 & 2.00 & 3.00 \\
\hline Troglodytes aedon parkmani & House Wren (in part) & VI & 7.10 & 8.28 & 3.00 & 5.25 \\
\hline T. aedon brunneicollis & House Wren (in part) & PR & 2.96 & 2.96 & 8.50 & 14.25 \\
\hline Thryomanes bewickii & Bewick's Wren & PR & 10.06 & 11.83 & 18.00 & 34.50 \\
\hline Campylorhynchus gularis & Spotted Wren & PR & 0.59 & 1.18 & NR & NR \\
\hline \multicolumn{7}{|l|}{ Regulidae } \\
\hline Regulus satrapa & Golden-crowned Kinglet & VI & 0.59 & 0.59 & NR & NR \\
\hline Regulus calendula & Ruby-crowned Kinglet & PR & 35.50 & 57.99 & 29.50 & 69.00 \\
\hline \multicolumn{7}{|l|}{ Turdidae } \\
\hline Myadestes occidentalis** & Brown-backed Solitaire & PR & 12.43 & 15.38 & 22.50 & 38.25 \\
\hline Catharus aurantiirostris & $\begin{array}{l}\text { Orange-billed Nightingale- } \\
\text { Thrush }\end{array}$ & PR & 0.59 & 0.59 & 4.00 & 7.00 \\
\hline Catharus occidentalis & Russet Nightingale-Thrush & PR & 5.92 & 6.51 & 12.00 & 23.25 \\
\hline Catharus guttatus & Hermit Thrush & VI & 0.59 & 1.18 & NR & NR \\
\hline Turdus assimilis & White-throated Thrush & PR & 0.59 & 0.59 & 3.00 & 4.75 \\
\hline Turdus migratorius & American Robin & PR & 33.14 & 56.80 & 33.00 & 86.25 \\
\hline \multicolumn{7}{|l|}{ Mimidae } \\
\hline Melanotis caerulescens & Blue Mockingbird & PR & 2.96 & 2.96 & 11.00 & 17.75 \\
\hline Toxostoma curvirostre & Curve-billed Thrasher & PR & 7.10 & 8.88 & 7.50 & 12.75 \\
\hline Mimus polyglottos & Northern Mockingbird & PR & NR & NR & 0.50 & 0.75 \\
\hline \multicolumn{7}{|l|}{ Ptiliogonatidae } \\
\hline Ptiliogonys cinereus & Gray Silky-flycatcher & PR & 25.44 & 99.41 & 37.50 & 163.50 \\
\hline \multicolumn{7}{|l|}{ Peucedramidae } \\
\hline Peucedramus taeniatus & Olive Warbler & PR & 18.93 & 21.30 & 13.00 & 19.50 \\
\hline \multicolumn{7}{|l|}{ Passeridae } \\
\hline Passer domesticus & House Sparrow & $\begin{array}{c}\mathrm{PR} / \\
\text { INTRO }\end{array}$ & 1.18 & 4.73 & 0.50 & 1.50 \\
\hline \multicolumn{7}{|l|}{ Fringillidae } \\
\hline Euphonia elegantissima & Elegant Euphonia & PR & NR & NR & 0.50 & 1.50 \\
\hline Haemorhous mexicanus & House Finch & PR & 16.57 & 52.07 & 19.50 & 78.75 \\
\hline Spinus pinus & Pine Siskin & PR & 4.14 & 8.88 & 3.00 & 9.75 \\
\hline Spinus psaltria & Lesser Goldfinch & PR & 15.98 & 52.07 & 15.50 & 90.25 \\
\hline \multicolumn{7}{|l|}{ Passerellidae } \\
\hline Arremon virenticeps & Green-striped Brushfinch & PR & NR & NR & 0.50 & 1.50 \\
\hline Atlapetes pileatus & Rufous-capped Brushfinch & PR & 2.96 & 3.55 & 3.50 & 9.00 \\
\hline Pipilo maculatus & Spotted Towhee & PR & 15.38 & 18.93 & 20.50 & 38.25 \\
\hline Aimophila rufescens & Rusty Sparrow & PR & 0.59 & 0.59 & 0.50 & 1.50 \\
\hline Melozone fusca & Canyon Towhee & PR & 15.38 & 33.73 & 20.50 & 44.75 \\
\hline Oriturus superciliosus & Striped Sparrow & $\mathrm{PR}$ & 5.33 & 14.79 & NR & NR \\
\hline
\end{tabular}




\begin{tabular}{|c|c|c|c|c|c|c|}
\hline \multirow{2}{*}{$\begin{array}{l}\text { Family } \\
\text { Species } \\
\end{array}$} & \multirow[t]{2}{*}{ Common name ${ }^{a}$} & \multirow{2}{*}{$\begin{array}{c}\text { Seasonal } \\
\text { status }^{\mathrm{b}}\end{array}$} & \multicolumn{2}{|c|}{$\begin{array}{c}\text { El Castillo } \\
\left(\mathrm{S}_{1} \text { Control site }\right) \\
\end{array}$} & \multicolumn{2}{|c|}{$\begin{array}{c}\text { Mina Dos Estrellas } \\
\left(\mathrm{S}_{2} \text { Mining site }\right)\end{array}$} \\
\hline & & & FRE $S_{1}{ }^{c}$ & $A B U S_{1}^{d}$ & FRE $S_{2}{ }^{\mathrm{c}}$ & $\mathrm{ABU} \mathrm{S_{2 } { } ^ { \mathrm { d } }}$ \\
\hline Spizella passerina & Chipping Sparrow & PR & 4.73 & 60.36 & 1.50 & 6.00 \\
\hline Spizella atrogularis & Black-chinned Sparrow & PR & 1.18 & 2.96 & NR & NR \\
\hline Melospiza melodia & Song Sparrow & PR & 1.18 & 1.78 & 2.50 & 5.25 \\
\hline Junco phaeonotus & Yellow-eyed Junco & PR & 15.38 & 34.32 & 22.00 & 64.50 \\
\hline \multicolumn{7}{|l|}{ Icteridae } \\
\hline Sturnella magna & Eastern Meadowlark & PR & 0.59 & 0.59 & NR & NR \\
\hline Icterus bullockii & Bullock's Oriole & PR & 2.96 & 4.14 & 1.50 & 3.75 \\
\hline Icterus abeillei & Black-backed Oriole & PR & NR & NR & 3.00 & 9.75 \\
\hline Icterus parisorum & Scott's Oriole & PR & 0.59 & 1.78 & 1.00 & 5.25 \\
\hline Molothrus aeneus & Bronzed Cowbird & PR & 0.59 & 0.59 & 0.50 & 1.50 \\
\hline Molothrus ater & Brown-headed Cowbird & PR & 0.59 & 2.37 & 0.50 & 0.75 \\
\hline \multicolumn{7}{|l|}{ Parulidae } \\
\hline Mniotilta varia & Black-and-white Warbler & VI & 1.78 & 1.78 & 1.00 & 1.50 \\
\hline Oreothlypis superciliosa & Crescent-chested Warbler & PR & 21.30 & 32.54 & 13.00 & 23.25 \\
\hline Oreothlypis celata & Orange-crowned Warbler & VI & 4.73 & 7.69 & 10.50 & 24.00 \\
\hline Oreothlypis crissalis & Colima Warbler & VI & NR & NR & 1.00 & 1.50 \\
\hline Oreothlypis ruficapilla & Nashville Warbler & VI & 0.59 & 0.59 & 0.50 & 0.75 \\
\hline Geothlypis tolmiei & MacGillivray's Warbler & VI & NR & NR & 0.50 & 0.75 \\
\hline Setophaga ruticilla & American Redstart & $\mathrm{TR}$ & 0.59 & 0.59 & NR & NR \\
\hline Setophaga coronata & Yellow-rumped Warbler & VI & 17.16 & 56.21 & 35.50 & 168.00 \\
\hline Setophaga graciae & Grace's Warbler & PR & NR & NR & 0.50 & 0.75 \\
\hline Setophaga nigrescens & Black-throated Gray Warbl. & VI & 1.78 & 1.78 & 2.50 & 3.75 \\
\hline Setophaga townsendi & Townsend's Warbler & VI & 14.20 & 33.73 & 15.50 & 49.50 \\
\hline Setophaga occidentalis & Hermit Warbler & VI & 9.47 & 17.75 & 5.00 & 11.25 \\
\hline Basileuterus rufifrons & Rufous-capped Warbler & PR & 2.37 & 2.96 & 4.50 & 9.50 \\
\hline Basileuterus belli & Golden-browed Warbler & PR & 4.73 & 7.10 & 1.50 & 3.00 \\
\hline Cardellina pusilla & Wilson's Warbler & VI & 5.92 & 5.92 & 7.50 & 12.00 \\
\hline Cardellina rubrifrons & Red-faced Warbler & VI & NR & NR & 1.00 & 2.25 \\
\hline Cardellina rubra & Red Warbler & PR & 2.37 & 3.55 & 2.50 & 5.25 \\
\hline Myioborus pictus & Painted Redstart & PR & 22.49 & 26.04 & 12.50 & 20.25 \\
\hline Myioborus miniatus & Slate-throated Redstart & PR & 2.37 & 3.55 & 1.00 & 1.50 \\
\hline \multicolumn{7}{|l|}{ Cardinalidae } \\
\hline Piranga flava & Hepatic Tanager & PR & 6.51 & 6.51 & 7.50 & 13.50 \\
\hline Piranga ludoviciana & Western Tanager & VI & 2.96 & 2.96 & 1.00 & 1.50 \\
\hline Piranga bidentata & Flame-colored Tanager & PR & 1.18 & 1.18 & 1.00 & 2.25 \\
\hline Pheucticus melanocephalus & Black-headed Grosbeak & PR & 27.81 & 38.46 & 18.50 & 37.50 \\
\hline Passerina caerulea & Blue Grosbeak & PR & 2.96 & 5.92 & 1.50 & 3.00 \\
\hline \multicolumn{7}{|l|}{ Thraupidae } \\
\hline Diglossa baritula & $\begin{array}{l}\text { Cinnamon-bellied } \\
\text { flowerpiercer }\end{array}$ & PR & 1.78 & 4.73 & 0.50 & 0.75 \\
\hline
\end{tabular}

(a) Common name according to AOS (2017). ${ }^{* *}$ Species under special protection (SEMARNAT 2010). (b) Seasonal status, PR: Permanent resident, VI: Winter visitant, TR: Transitory, INTRO: Introduced. (c) FRE = frequency (probability of presence in point counts; (d) ABU = relative abundance expressed in number of individuals in 100 point counts; (e) NR = Non recorded species. 
Psaltriparus minimus (Bushtit) and Spinus psaltria (Lesser Goldfinch) (Table 1). On the other hand, the species with the highest frequency of occurrence in $S_{1}$ were $H$. leucotis, $R$. calendula, T. migratorius, $P$. cinereus and Myioborus pictus (Painted Redstart); while in $\mathrm{S}_{2}$, the most frequent species were $H$. leucotis, $P$. cinereus, $S$. coronata, R. calendula, and Myadestes occidentalis (Brown-backed Solitaire) (Table 1).

The Mann-Whitney U test showed no significant differences in the average number of species and individuals per count between sites $(P>0.1)$. However, there were significant differences in the abundance of 18 species (Table 2). In reference to the similarity between sites, the Sorensen index revealed $85 \%$ qualitative similarity, while the Morisita-Horn index showed 93\% quantitative similarity.

The Analysis of Similarity (ANOSIM) indicated a high level of correspondence between both communities $(R=0.0445, P=0.0001)$. On the other hand, the SIMPER analysis suggested that the few extant differences between them are attributable to 18 species (Table 3), which add up to $62 \%$ of the differences between sites. The SIMPER test gives greater weight to abundance, such that species contributing to the differences between communities are those with the highest number of records. Overall the bird communities at the study sites were very similar to one another (as it was also evident with ANOSIM), although some species had clear differences in their abundance in both sites (Table 2), such as $S$. coronata, $M$. caerulescens, $O$. superciliosus, C. auratus, and T. mexicanus.

\section{DISCUSSION}

Despite the fact that the OTMD has historically been very important for its economic prosperity, biological inventories in the area are virtually non-existent. This work provides the first bird species inventory for Mina Dos Estrellas and Tlacotepec, with 108 species (19.7\% of those registered in the state of Michoacan) (VillaseñorGómez 2005), and $83 \%$ of the species recorded from Sierra Chincua at MBBR (SEMARNAT 2001), the nearest area with available ornithological information. According to the NOM-059 (SEMARNAT 2010), two species are under special protection: Accipiter cooperi (Cooper's Hawk) and M. occidentalis.

After 65 years of the cessation of extractive mining activities, bird communities at the OTMD have a high degree of similarity (85\% qualitative and 93\% quantitative), a pattern that coincides with the results

Table 2. Mean relative abundance of the species with significant differences in abundance between study sites in El OroTlalpujahua, Mining District, during 2014-2015.

\begin{tabular}{|c|c|c|c|c|c|}
\hline Species & $\begin{array}{l}\text { Individuals } \\
\text { recorded }\end{array}$ & $\begin{array}{l}\text { Feeding } \\
\text { guild }^{\mathrm{a}}\end{array}$ & $\begin{array}{l}\text { Mean Control Site, } \\
\text { El Castillo } S_{1}(E E)\end{array}$ & $\begin{array}{c}\text { Mean Tilings site, } \\
\text { Mina Dos Estrellas } S_{2}(E E)\end{array}$ & $P$ \\
\hline Setophaga coronata & 145 & Omn & $0.237(0.044)$ & $0(0)$ & $0.001^{* *}$ \\
\hline Myadestes occidentalis & 75 & Fru & $0.147(0.032)$ & $0.25(0.034)$ & $0.014^{*}$ \\
\hline Sitta carolinensis & 69 & Gra & $0.266(0.042)$ & $0.120(0.023)$ & $0.008^{* *}$ \\
\hline Myioborus pictus & 68 & Ins & $0.248(0.037)$ & $0.130(0.024)$ & $0.010^{*}$ \\
\hline Oreothlypis superciliosa & 67 & Ins & $0.230(0.036)$ & $0.140(0.026)$ & $0.045^{*}$ \\
\hline Melanerpes formicivorus & 61 & Omn & $0.094(0.022)$ & $0.225(0.035)$ & $0.010^{*}$ \\
\hline Thryomanes bewickii & 56 & Ins & $0.100(0.023)$ & $0.195(0.03)$ & $0.028^{*}$ \\
\hline Catharus occidentalis & 40 & Fru & $0.065(0.020)$ & $0.145(0.03)$ & $0.043^{*}$ \\
\hline Oreothlypis celata & 33 & Ins & $0.059(0.021)$ & $0.115(0.024)$ & $0.043^{*}$ \\
\hline Melanotis caerulescens & 28 & Ins/Fru & $0.029(0.013)$ & $0.115(0.023)$ & $0.003^{* *}$ \\
\hline Troglodytes a. brunneicollis. & 22 & Ins & $0.029(0.013)$ & $0.085(0.020)$ & $0.025^{*}$ \\
\hline Cyanocitta stelleri & 16 & Omn & $0.076(0.027)$ & $0.015(0.011)$ & $0.015^{*}$ \\
\hline Tyrannus vociferans & 15 & Ins & $0.065(0.020)$ & $0.020(0.010)$ & $0.049^{*}$ \\
\hline Oriturus superciliosus & 12 & Omn & $0.071(0.025)$ & $0(0)$ & $0.001^{* *}$ \\
\hline Catharus aurantiirostris & 10 & Fru & $0.005(0.005)$ & $0.045(0.016)$ & $0.035^{*}$ \\
\hline Colaptes auratus & 10 & Gra/Ins & $0.053(0.017)$ & $0.005(0.005)$ & $0.005^{* *}$ \\
\hline Trogon mexicanus & 10 & Ins/Fru & $0.059(0.024)$ & $0(0)$ & $0.004^{* *}$ \\
\hline Icterus abeillei & 6 & Ins & $0(0)$ & $0.030(0.012)$ & $0.023^{*}$ \\
\hline
\end{tabular}

(a) Fru: Frugivorous; Gra: Granivorous; Ins: Insectivorous; Omn: Omnivorous.

Non-parametric Mann-Whitney $U$ tests: ${ }^{*} P<0.5$ and $>0.1,{ }^{* *} P<0.01$; SE $=$ Standard error. 
Table 3. Contribution of the bird species to the differences between the communities at the control site and a mining site at El Oro-Tlalpujahua Mining District, central Mexico.

\begin{tabular}{lcccc}
\hline Species & $\begin{array}{c}\text { Contribution (\%) } \\
\text { to the difference }\end{array}$ & $\begin{array}{c}\text { Cumulative } \\
\text { percentage of the } \\
\text { difference }\end{array}$ & $\begin{array}{c}\text { Species abundance } \\
\text { per count at the } \\
\text { control site }\end{array}$ & $\begin{array}{c}\text { Species abundance } \\
\text { per count at the } \\
\text { mining site }\end{array}$ \\
\hline Hylocharis leucotis & 6.79 & 6.79 & 0.75 & 0.811 \\
Setophaga coronata & 5.18 & 11.97 & $\mathbf{0 . 5 2 5}$ & $\mathbf{0 . 2 3 7}$ \\
Turdus migratorius & 5.16 & 17.12 & 0.405 & 0.438 \\
Ptiliogonys cinereus & 4.96 & 22.08 & 0.465 & 0.325 \\
Regulus calendula & 4.53 & 26.61 & 0.32 & 0.432 \\
Pheucticus melanocephalus & 3.50 & 30.11 & 0.21 & 0.325 \\
Haemorhous mexicanus & 3.18 & 33.29 & 0.22 & 0.237 \\
Junco phaeonotus & 3.12 & 36.41 & 0.23 & 0.195 \\
Melozone fusca & 3.02 & 39.42 & 0.215 & 0.219 \\
Myadestes occidentalis & 2.83 & 42.26 & 0.25 & 0.148 \\
Spinus psaltria & 2.77 & 45.03 & 0.18 & 0.195 \\
Pipilo maculatus & 2.63 & 47.66 & 0.23 & 0.172 \\
Sitta carolinensis & 2.59 & 50.25 & 0.12 & 0.266 \\
Myioborus pictus & 2.50 & 52.75 & 0.13 & 0.249 \\
Oreothlypis superciliosa & 2.47 & 55.22 & 0.14 & 0.231 \\
Setophaga townsendi & 2.42 & 57.64 & 0.195 & 0.148 \\
Melanerpes formicivorus & 2.30 & 59.94 & $\mathbf{0 . 2 2 5}$ & $\mathbf{0 . 0 9 4 7}$ \\
Peucedramus taeniatus & 2.22 & 62.16 & 0.13 & 0.195 \\
\hline
\end{tabular}

of other studies. In southern Spain, at the Guadiamar corridor, a severely contaminated environment at the Aznalcollar mine, in restoration since 2000, MárquezFerrando (2008) found that the composition of bird communities exposed to mining waste remnants after eight years of abandonment was $80 \%$ similar to those at natural sites without exposure to mining wastes. Similarly, Osipov \& Biserov (2017) studied the succession of bird communities in a Boreal Mountain-Valley landscape disturbed by gold mining in the Niman River (at the Bureya mountains, Russia); their findings indicate that sites with tailings 35-40 years after abandonment were similar in species composition to areas of valleys without disturbance, even though density of species was lower in the mining sites. On the other hand, abandoned tailing sites had a more complex successional vegetation and their communities of birds were more diverse and abundant, as were the mountain forest communities without disturbance. Nichols \& Watkins (1984) and Armstrong $\&$ Nichols (2000) studied the avifaunal recolonization of rehabilitated bauxite mines in the Jarrah Forest of southwestern Australia. They compared bird communities in a forest where extraction started in 1963 in rehabilitated
Eucalyptus (Eucalyptus marginata) forest, and found that avian communities were very similar after a period of 24 and 30 years, and that the bird communities could reach up to $65 \%$ similarity within the first $4-5$ years of abandonment, and $73.5 \%$ similarity after 16-17 years. According to this, similarity of communities increases with time in disturbed environments, where natural regeneration or restoration processes have taken place.

The area of OTMD has gone through a process of natural regeneration, in which those plant species most tolerant to disturbance, and/or those that were exploited to a lesser extent, reestablished the vegetation on the area, and its composition and structure support very similar bird communities. The reestablishment of forests with similar physiognomy in the study site may indicate the presence of suitable resources that maintain similar bird communities at both sites (McArthur \& McArthur 1961). However, differences in the abundance of 18 species also suggests the existence of specific effects. They might be related to the sensitivity to pollutants, differences in the availability of specific resources (e.g., food, breeding sites, feeding territories, perching structures), or other factors not taken into account (Loyn 1985, Gould \& Mackey 
2015). For example, differences found in $M$. formicivorus might reflect the presence of tall eucalyptus trees and snags at the tailings site (Mina Dos Estrellas), where most individuals were recorded. As suggested, food resources, perching structures, and breeding sites can be some of the primary limiting factors in the species distributions and preferences within a given habitat (Cody 1985, Hutto 1985, Jones 2001).

Studies on the effects of secondary succession in forests have shown that in general, early successional bird communities include more generalist granivorous, omnivorous and insectivorous species, considered as pioneer species indicative of disturbance (Rangel-Salazar et al. 2009, Becker et al. 2013). As succession progresses, structural diversity of vegetation increases and, depending on the community composition, specialized frugivorous, nectarivorous, and specialized insectivorous species (soil, bark, understory and foliage gleaning) colonize the habitat and increase in numbers (May 1982, Winkler 2005, Rangel-Salazar et al. 2009). Becker et al. (2013) found that bird communities in restored mining areas in southern Brazil had similar species richness between sites after 10-20 years of abandonment, although differences in the abundance of species according to feeding guild were evident: granivorous species decreased, whereas carnivorous, frugivorous, and nectarivorous increased (especially those forests dependent species); omnivorous species remained stable. Their findings suggest that effects could be reflected through changes in functional groups, or can be species specific. In the case of OTMD, some generalists, omnivorous and ground insectivorous species (M. formicivorus, S. coronata, J. phaeonotus and O. celata) were significantly more abundant in the tailings site, meanwhile at the control site more specialized species such as nectarivorous and bark insectivores prevailed (H. leucotis, R. calendula, S. carolinensis, M. pictus and $O$. superciliosa). Further study is needed to determine whether these differences are attributable to the existence of remnant mining effects after 65 years of abandonment.

Dendrochronological analysis of trees at MBBR suggested that individuals of Sacred Fir (Abies religiosa) are 106 years old, and those of Smooth-bark Mexican Pine (Pinus pseudostrobus) are 120 years old. Evidence indicates that the MBBR region has also been subjected to historical disturbance regimes caused by logging (Sáenz-Ceja 2015). The presence of old railway tracks in the core zone of MBBR at Sierra Chincua supports the idea of active logging in the past. Probably, regeneration processes took place almost at the same time, and, as such, the similarities in avian communities between MBBR and OTMD may reflect the effects of succession in a wider geographic area. We suggest that OTMD represents an important habitat for resident and migratory bird species in the surroundings of MBBR.
Information on remnant effects of abandoned mines is very scarce. It might prove useful to explore their effects on animal communities under scenarios of revegetation (natural succession) or restoration. Current information on mining impacts refers mostly to the response of biotic communities in active mining districts, where negative effects have been found on birds, rodents, and vegetation. In vertebrates, high concentrations of toxic elements are present in kidney and liver tissues, and they relate to their concentrations in the plants on which they feed (EspinosaReyes et al. 2014). Bioaccumulation of these elements is known to cause negative effects at the neurological (lethargy), physiological (chronic stress and changes in DNA structure), behavioral (decrease of appetite), and reproductive level (low production of eggs in birds) (Festa et al. 2003, Seewagen 2009, Chapa-Vargas et al. 2010), contributing to the decline of biodiversity at contaminated sites. The rivers of Santa Cruz, San Pedro and Colorado in Sonora, Mexico, which receive flows from copper mines, are very important sites for breeding and wintering birds, despite the negative impacts of their low water quality (Sprouse 2005, Villaseñor-Gómez 2006); they have not been studied in detail. Little is known on the effects of potentially toxic elements in tailing residual soils and their bioavailability. It would be necessary to study tailing chemistry, the exposure paths for those elements, and bio-magnification effects on functional groups or specific bird species that may be affected at the physiological level (Hudson \& Bouwman 2008).

While the establishment of the DMOT historically generated a significant disturbance in the ecosystem, 65 years after the end of its activities current conditions seem favorable for the maintenance of avian communities as a major component of the regional biodiversity, since differences at the community level were not remarkable. Although it is not possible to assess the intensity of the environmental effects caused by mining at DMOT in the past, modification of the natural environment has left permanent traces, such as soil derived from tailings and the absence of some tree species (such as Sacred Fir, $A$. religiosa, present in neighboring forests) that were not able to cope with changes. The relative geochemical stability and the revegetation of tailings may indicate the existence of low intensity impacts at the present. Although there is no evidence at this time, the bioavailability of some potentially toxic elements could trigger processes of biomagnification in some species, inducing negative health effects on bird individuals in the region. Therefore, it is important to continue working on this subject and to analyze some physiological indicators of performance (such as robustness, condition index, fat reserves, and the Heterophile/Lymphocyte Index) in local birds, in order to evaluate their health and fitness. We also suggest to gain further insights on the role of vegetation structure, 
functional responses of communities (through feeding guilds), and the current anthropogenic impacts that may be influencing bird communities.

\section{ACKNOWLEDGEMENTS}

This paper is part of the MSc. Dissertation of K.I. Lemus-Ramírez (Grant 540656, CONACyT-Mexico). We recognize the financial support from Universidad Michoacana de San Nicolás de Hidalgo (Coordinación de Investigación Científica, Project "Avifauna de la región Tlalpujahua-El Oro: estructura de comunidades, uso de hábitat y expresión de estrés ambiental (2014-2018)". We are grateful to the staff of Mina Dos Estrellas (Museo Técnico Minero Siglo XIX) and Ms. G. Duarte-Godinez for the permits to work on their property; R.A. Medina Nieves and J.C. Perez Magaña supported us during field activities. We also appreciate the effort and dedication of anonymous reviewers who helped to improve this work.

\section{REFERENCES}

Altaf M., Javid A., Khan A.M., Khan M.S.H., Umair M. \& Ali Z. 2018. Anthropogenic impact on the distribution of the birds in the tropical thorn forest, Punjab, Pakistan. Journal of Asia-Pacific Biodiversity 11: 229-236.

AOS [American Ornithological Society]. 2017. American Ornithological Society's check-list of North American birds. http:// www.americanornithology.org/content/checklist-north-andmiddle-american-birds.

Armstrong K.N. \& Nichols O.G. 2000. Long term trends in avifaunal recolonisation of rehabilitated bauxite mines in the Jarrah Forest of south-western Australia. Forest Ecology and Management 126: 213-225.

Badii M.H., Landeros J. \& Cerna E. 2007. Patrones de asociación de especies y sustentabilidad. International Journal of Good Conscience 3: 632-660.

Balistrieri L.S., Box S.E., Bookstrom A.A., Hooper R.L. \& Mahoney J.B. 2002. Impacts of historical mining in the Coeur d'Alene River Basin, p. 1-34. In: Balistrieri L.S. \& Stillings L.L. (eds.). Pathways of metal transfer from mineralized sources to bioreceptors: a synthesis of the mineral resources program's past environmental studies in the western United States and future research directions. Washington: U.S. Geological Survey.

Balmer O. 2002. Species list in ecology and conservation: abundances matter. Conservation Biology 16: 1160-1161.

Batty L.C. 2005. The potential importance of mine sites for biodiversity. Mine Water and the Environment 24: 101-103.

Becker R.G., Paise G. \& Pizo M.A. 2013. The structure of bird communities in areas revegetated after mining in south Brazil. Revista Brasileira de Ornitologia 21: 221-234.

Blake J.G. 2007. Neotropical forest bird communities: a comparison of species richness and composition at local and region scales. Condor 109: 237-255.

Boulet M.P. \& Larocque A.C.L. 1988. A comparative mineralogical and geochemical study of sulfide mine tailings at two sites in New Mexico, U.S.A. Environmental Geology 33: 130-142.

Buskirk W.H. \& McDonald J.L. 1995. Comparison of point count sampling regimes for monitoring forest birds, p. 25-34. In: Ralph
C.J., Sauer J.R \& Droege S. (eds.). Monitoring bird populations by point counts. Albany: Pacific Southwest Research Station.

Cody M.L. 1985. Habitat selection in birds. Orlando: Academic Press.

Colwell R.K. 2013. EstimateS, version 9.1.0: statistical estimation of species richness and shared species from samples. http://purl.oclc.org/ estimates (Access on 05 August 2019).

Colwell R.K. \& Coddington J.A. 1994. Estimating terrestrial biodiversity through extrapolation. Philosophical Transactions of the Royal Society of London B: Biological Sciences 345: 101-118.

Corona-Chávez P. \& Uribe-Salas J.A. 2009. Atlas cartográfico del Distrito Minero El Oro-Tlalpujahua. Morelia: Universidad Michoacana de San Nicolás de Hidalgo.

Corona-Chávez P., Uribe-Salas J.A., Razo-Perez N., Martínez-Medina M., Maldonado-Villanueva R., Ramos-Arroyo Y.R. \& RoblesCamacho J. 2010. The impact of miningin the regional ecosystem: the Mining District of El Oro and Tlalpujahua, México. De Re Metallica 15: 21-34.

Coronado-Martínez Y. 2016. El ecoturismo como opción para el desarrollo local sustentable en el Pueblo Mágico de Tlalpujahua, Michoacán. MSc. Dissertation. Ciudad de México: Instituto Politécnico Nacional.

Cristol D.A., Brasso R.L., Condon A.M., Fovargue R.E., Friedman S.L., Hallinger K.K., Monroe A.P. \& White A.E. 2008. The movement of aquatic mercury through terrestrial food webs. Science 320: 335.

Chao A., Chazdon R.L., Colwell R.K. \& Shen T.J. 2005. A new statistical approach for assessing compositional similarity based on incidence and abundance data. Ecology Letters 8: 148-159.

Chapa-Vargas L., Mejía-Saavedra J., Monzalvo-Santos K. \& PueblaOlivares F. 2010. Blood lead concentrations in wild birds from a polluted mining region at Villa de la Paz, San Luis Potosi, Mexico. Journal of Environmental Science and Health A 45: 90-98.

Eagles-Smith C.A., Wiener J.G., Eckley C.S., Willacker J.J., Evers D.C., Marvin-DiPasquale M., Obrist D., Fleck J.A., Aiken G.R., Lepak J.M., Jackson A.K., Webster J.P., Stewart A.R., Davis J.A., Alpers C.N. \& Ackerman J.T. 2016. Mercury in western North America: a synthesis of environmental contamination, fluxes, bioaccumulation, and risk to fish and wildlife. Science of the Total Environment 568: 1213-1226.

Edwards D.P., Larsen T.H., Docherty T.D.S., Ansell F.A., Hsu W.W., Derhé M.A., Hamer K.C. \& Wilcove D.S. 2011. Degraded lands worth protecting: the biological importance of southeast Asia's repeatedly logged forests. Proceedings of the Royal Society of London B: Biological Sciences 278: 82-90.

Eisler R. 2004. Mercury hazards from gold mining to humans, plants, and animals. Reviews of Environmental Contamination and Toxicology 181: 139-198.

Espinosa-Reyes G., González-Mille D.J., Ilizaliturri-Hernández C.A., Mejía-Saavedra J., Cilia-López V.G., Costilla-Salazar R. \& DíazBarriga F. 2014. Effect of mining activities in biotic communities of Villa de la Paz, San Luis Potosi, Mexico. BioMed Research International 2014: 1-13.

Festa F., Cristaldi M., Ieradi L.A., Moreno S. \& Cozzi R. 2003. The comet assay for the detection of DNA damage in Mus spretus from Donana National Park. Environmental Research 91: 54-61.

Garitano-Zavala A., Cotín J., Borràs M. \& Nadal J. 2010. Trace metal concentrations in tissues of two tinamou species in mining areas of Bolivia and their potential as environmental sentinels. Environmental Monitoring and Assessment 168: 629-244.

Gonçalves-Rodriguez G. \& Shraft B.W. 2001. Review of benthic invertebrate fauna in extremely acidic environments $(\mathrm{pH} \leq 3)$. Mine Water and the Environment 20: 114-121.

Gould S.F. \& Mackey B.G. 2015. Site vegetation characteristics are more important than landscape context in determining bird assemblages in revegetation. Restoration Ecology 23: 670-680.

Howell S.N.G. \& Webb S. 2005. A guide to the birds of Mexico and north Central America. New York: Oxford University Press. 
Hudson A. \& Bouwman H. 2008. Birds associated with a tailings storage facility and surrounding areas from a South African gold mine. African Journal of Ecology 46: 276-281.

Hutto R.L. 1985. Habitat selection by nonbreeding, migratory land birds, p. 455-476. In: Cody M.L. (ed.). Habitat selection in birds. Orlando: Academic Press.

Hutto R.L., Pletschet S.M. \& Hendricks P. 1986. A fixed-radius point count method for nonbreeding and breeding season use. $A u k 103$ : 593-602.

INEGI [Instituto Nacional de Estadística, Geografía e Informática]. 2009. Prontuario de información geográfica municipal de los Estados Unidos Mexicanos: clave geoestadística 16093. http://www3.inegi.org.mx/sistemas/mexicocifras/datosgeograficos/16/16093.pdf. (Access on 10 January 2016).

Jacob D.L. \& Otte M.L. 2004. Long-term effects of submergence and wetland vegetation on metals in a 90-year old abandoned $\mathrm{Pb}-\mathrm{Zn}$ mine tailings pond. Environmental Pollution 130: 337-345.

Jones J. 2001. Habitat selection studies in avian ecology: a critical review. Auk 118: 557-562.

Kossoff D., Dubbin W.E., Alfredsson M., Edwards S.J., Macklin M.G. \& Hudson-Edwards K.A. 2014. Mine tailings dams: characteristics, failure, environmental impacts, and remediation. Applied Geochemistry 51: 229-245.

Lacerda L.D. 1997. Global mercury emissions from gold and silver mining. Water, Air, \& Soil Pollution 97: 209-221.

Lefcort H., Meguire R.A., Wilson L.H. \& Ettinger W.F. 1988. Heavy metals alter the survival, growth, metamorphosis, and antipredatory behavior of Columbia Spotted Frog (Rana luteiventris) tadpoles. Archives of Environmental Contamination and Toxicology 35: 447-456.

Lock K., Janssens F. \& Janssen C.R. 2003. Effects of metal contamination on the activity and diversity of springtails in an ancient $\mathrm{Pb}-\mathrm{Zn}$ mining area at Plombières, Belgium. European Journal of Soil Biology 39: 25-29.

Loyn R.H. 1985. Bird population in successional forest of Mountain Ash Eucalyptus regnans in central Victoria. Emu 85: 213-231.

Mahmoud S.H. \& Gan T.Y. 2018. Impact of anthropogenic climate change and human activities on environment and ecosystem services in arid regions. Science of the Total Environment 633: 1329-1344.

Manson R.H. \& Jardel-Peláez E.J. 2009. Perturbaciones y desastres naturales: impactos sobre las ecorregiones, la biodiversidad y el bienestar socioeconómico, p. 131-184. In: Sarukán J. (ed.). Capital Natural de México, v. 2: estado de conservación y tendencias de cambio. Ciudad de México: CONABIO.

Márquez-Ferrando R. 2008. Las comunidades de aves y reptiles del corridor Verde del Guadiamar después del vertido minero de Aznalcóllar. MSc. Dissertation. Granada: Universidad de Granada.

May P.G. 1982. Secondary succession and breeding bird community structure: patterns of resource utilization. Oecologia 55: 208-216.

McArthur R.H. \& McArthur J.W. 1961. On bird species diversty. Ecology 42: 594-598.

Muñiz-Castro M.A. 2008. Sucesión secundaria y establecimiento de especies arbóreas nativas para restauración de Bosque Mesófilo de Montaña en potreros abandonados del centro de Veracruz. Ph.D. Thesis. Xalapa: Instituto de Ecología A.C.

Nichols O.G. \& Watkins D. 1984. Bird utilization of rehabilitated bauxite minesites in western Australia. Biological Conservation 30: 109-131.

Nieto-Monroy A.P. 2007. Variabilidad espacial y temporal de la Hidrogeoquimica de arroyos del Distrito Minero El Oro-Tlalpujahua. Ph.D. Thesis. Guanajuato: Universidad de Guanajuato.

O'Sullivan A.D., McCabe O.M., Murray D.A. \& Otte M.L. 1999. Wetlands for rehabilitation of metal mine wastes. Proceedings of the Royal Irish Academy B: Biology and Environment 99: 11-17.
Osipov S.V. \& Biserov M.F. 2017. Population of birds in the Boreal Mountain-Valley landscape disturbed by gold mining. Russian Journal of Ecology 48: 45-50.

Osuna-Vallejo V., Lindig-Cisneros R.A., Sáenz-Romero C. \& Cruzde-León J. 2016. Ensayo de mesocosmos especies y procedencias de coníferas en residuos mineros de Tlalpujahua, Michoacán. XX Congreso Mexicano de Botánica. Ciudad de México: Sociedad Botánica de México.

Ouboter P.E., Landburg G.A., Quik J.H.M., Mol J.H.A. \& van del Lugt F. 1999. Mercury levels in pristine and gold mining impacted aquatic ecosystems of Suriname, South America. AMBIO 41: 873-882.

Pérez-Tris J. 1999. El peso de las aves en paso: ¿Una medida de condición física o acumulación de grasa? Revista de Anillamiento 3: $11-15$.

Perotti M., Petrini R., D'Orazio M., Ghezzi L., Giannecchini R. \& Vezzoni S. 2017. Thallium and other potentially toxic elements in the Baccatoio stream catchment (northern Tuscany, Italy) receiving drainages from abandoned mines. Mine Water and the Environment 37: 1-11.

Pickett S.T.A. \& White P.S. 1985. The ecology of natural disturbance and patch dynamics. Cambridge: Academic Press.

Ramírez-Ramírez M.I. 2001. Los espacios forestales de la Sierra de Angangueo (estados de Michoacán y México), México: una revisión geográfica. Ph.D. Thesis. Madrid: Universidad Complutense de Madrid.

Rangel-Salazar J.L., Enríquez P.L. \& Sántiz-López E.C. 2009. Variación de la diversidad de aves de sotobosque en el Parque Nacional Lagos de Motebello, Chiapas, México. Acta Zoológica Mexicana 25: 479-495.

Rösner U. 1988. Effects of historical mining activities on surface water and groundwater: an example from northwest Arizona. Environmental Geology 33: 224-230.

Rubio I., Martinez-Madrid M., Mendez-Fernandez L., Galarza A. \& Rodriguez P. 2016. Heavy metal concentration in feathers of Little Egret (Egretta garzetta) nestlings in three coastal breeding colonies in Spain. Ecotoxicology 25: 30-40.

Sáenz-Ceja J.E. 2015. Recostrucción dendrocronológica de la historia de establecimiento de Pinus pseudostrobus y Abies religiosa en la Reserva de la Biosfera Mariposa Monarca. Ciudad de México: Instituto de Investigaciones en Ecosistemas y Sustentabilidad.

Seewagen C.L. 2009. Threats of environmental mercury to birds: knowledge gaps and priorities for future research. Bird Conservation International 20: 112-123.

SEMARNAT [Secretaría de Medio Ambiente y Recursos Naturales]. 2001. Programa de manejo de la Reserva de la Biosfera Mariposa Monarca. Ciudad de México: CONANP.

SEMARNAT [Secretaría de Medio Ambiente y Recursos Naturales]. 2010. Norma Oficial Mexicana NOM-059-SEMARNAT-2010 de 30 de Diciembre del 2010. http://www.profepa.gob.mx/ innovaportal/file/435/1/NOM_059_SEMARNAT_2010.pdf (Access on 02 April 2017).

Sprouse T.W. 2005. Water issue on the Arizona-Mexico border. Tucson: Water Resources Research Center.

Struckhoff M.A., Stroh E.D. \& Grabner K.W. 2013. Effects of miningassociated lead and zinc soil contamination on native floristic quality. Journal of Environmental Management 119: 20-28.

Ugalde-Lezama S., Alcántara-Carbajal J.L., Tarango-Arámbula L.A., Ramírez-Valverde G. \& Mendoza-Martínez G.D. 2012. Fisonomía vegetal y abundancia de aves en un bosque templado con dos niveles de perturbación en el Eje Neovolcánico Transversal. Revista Mexicana de Biodiversidad 83: 133-143.

Ventakeswarlu K., Nirola R., Kuppusamy S., Thavamani P., Naidu R. \& Megharaj M. 2016. Abandoned metalliferous mines: ecological impacts and potential approaches for reclamation. Reviews in Environmental Science and Bio/Technology 15: 327-354. 
Villaseñor-Gómez L.E. 2005. Aves, p. 101-103. In: Villaseñor-Gómez L.E. \& Leal-Nares O.A. (eds.). La biodiversidad en Michoacán. Estudio de estado. Ciudad de México: Comisión Nacional para el Conocimiento y Uso de la Biodiversidad.

Villaseñor-Gómez J.F. 2006. Habitat use and the effects of disturbance on wintering birds using riparian habitats in Sonora, Mexico. Ph.D. Thesis. Missoula: University of Montana.

Winkler D. 2005. Ecological succession of breeding bird communities in deciduous and coniferous forest in the Sopron Mountains, Hungary. Acta Silvatica et Lignaria Hungarica 1: 49-58.

Xu X., Xie Y., Qi K., Luo Z. \& Wang X. 2018. Detecting the response of bird communities and biodiversity to habitat loss and fragmentation due to urbanization. Science of the Total Environment 624: 1561-1576.

Associate Editor: Marcos P. Dantas. 